\title{
Formation and enhanced biocidal activity of water-dispersable organic nanoparticles
}

\author{
HAIFEI ZHANG ${ }^{1}$, DONG WANG ${ }^{2}$, RACHEL BUTLER ${ }^{1}$, NEIL L. CAMPBELL ${ }^{1}$, JAMES LONG ${ }^{2}$, BIEN TAN ${ }^{1}$, \\ DAVID J. DUNCALF ${ }^{2,3}$, ALISON J. FOSTER ${ }^{2,3}$, ANDREW HOPKINSON ${ }^{3}$, DAVID TAYLOR ${ }^{3}$, DORIS ANGUS ${ }^{2}$, \\ ANDREW I. COOPER ${ }^{1 *}$ AND STEVEN P. RANNARD ${ }^{1,2,3 *}$
}

\author{
'Department of Chemistry, University of Liverpool, Crown Street, Liverpool L69 3BX, UK \\ ${ }^{2}$ IOTA NanoSolutions Ltd, Crown Street, Liverpool L69 7ZB, UK \\ ${ }^{3}$ Unilever R\&D, Port Sunlight Laboratories, Quarry Road East, Bebington, Wirral CH63 3JW, UK \\ *e-mail: srannard@liv.ac.uk
}

\begin{abstract}
Water-insoluble organic compounds are often used in aqueous environments in various pharmaceutical and consumer products. To overcome insolubility, the particles are dispersed in a medium during product formation ${ }^{1}$, but large particles that are formed may affect product performance and safety. Many techniques have been used to produce nanodispersions-dispersions with nanometre-scale dimensions-that have properties similar to solutions $^{2-4}$. However, making nanodispersions requires complex processing, and it is difficult to achieve stability over long periods ${ }^{1}$. Here we report a generic method for producing organic nanoparticles with a combination of modified emulsiontemplating ${ }^{5}$ and freeze-drying. The dry powder composites formed using this method are highly porous, stable and form nanodispersions upon simple addition of water. Aqueous nanodispersions of Triclosan (a commercial antimicrobial agent) produced with this approach show greater activity than organic/aqueous solutions of Triclosan.
\end{abstract}

As the range of nanoparticle-based technologies increases, issues of sustainability and environmental impact become more important. Standard guidelines for evaluating long-term chemical risks/benefits, such as environmental persistence, bioaccumulation and nanoparticle toxicity have been suggested recently ${ }^{6}$. In general, insoluble inorganic nanoparticles are expected to be more environmentally persistent than organic nanomaterials, because even poorly soluble organic compounds are ultimately completely soluble in water.

Drug solubility controls circulating plasma levels, pharmacokinetics and bioavailability of orally-dosed particulate pharmaceuticals that are poorly water soluble ${ }^{2}$. Although medical applications have received considerable attention, significant opportunities still remain for nanodispersions of other commercial low-solubility organic molecules, especially as their environmental fate is well understood.

Aqueous nanodispersions offer the benefits of dispersing poorly water-soluble molecules without directly using organic solvents. Although many organic nanoparticle production methods exist ${ }^{3,4}$, few are truly generic approaches. For example, wet-milling is time- and energy-intensive, transforming bulk organic materials into nanoparticles ${ }^{3}$, and is incompatible with explosive, low-melting, or temperature-sensitive compounds. Moreover, 'milling agent' degradation introduces impurities, and producing uniform organic nanoparticles with diameters $<300 \mathrm{~nm}$ is difficult owing to particle aggregation ${ }^{7}$.

Horn and Rieger ${ }^{4}$ comprehensively reviewed the main classes of solvent-based nanoparticle formation routes, described mainly as 'precipitation and condensation processes'. Our new route to aqueous nanodispersions differs from these classifications and other techniques including salting-out ${ }^{8}$, film rehydration ${ }^{9,10}$ and vesicle formation ${ }^{11}$. We use steps used previously to produce so-called 'emulsion-templated'12 or 'polyHIPE' polymers ${ }^{5}$ without using chemical crosslinking to maintain the porous structure. Initially, we generate an oil-in-water $(\mathrm{O} / \mathrm{W})$ emulsion using a volatile organic solvent oil phase containing a dissolved organic compound, and a continuous aqueous phase containing a stabilizer or mixture of stabilizers (for example, water-soluble polymers or surfactants). The emulsion is dripped or atomized onto the surface of a cryogenic liquid, or directly frozen, resulting in the formation of frozen beads $(2-3 \mathrm{~mm}$ diameter), micrometresized powders or large monolithic structures. Finally, both the water and the organic solvent are removed by freeze-drying, generating dry composite materials comprising the water-insoluble organic compound and water-soluble polymers/surfactants. The highly porous composites dissolve readily in water, releasing the organic compound as nanoparticulate dispersions, which resemble transparent molecular solutions (Fig. 1a-c).

The process was exemplified using a model water-insoluble compound, Oil Red (OR) dissolved in cyclohexane $^{13}(\mathrm{CH}$, $0.018 \mathrm{wt} \%$ ), and poly(vinyl alcohol) (PVA) and sodium dodecyl sulphate (SDS) dissolved in water $(\mathrm{O} / \mathrm{W}$ ratio $=75: 25$; $5 \mathrm{wt} \% \mathrm{PVA}, 5 \mathrm{wt} \% \mathrm{SDS})$. The porous emulsion-templated structures (Fig. 1d-e) and spheroidal OR nanoparticles (Fig. 1f) were revealed by scanning electron microscopy (SEM) and scanning transmission electron microscopy (STEM) (Fig. 1g). The materials dissolve rapidly in water, producing clear red OR nanodispersions, visually identical to solutions (see Supplementary Information, Movie S1). Analysis of the nanodispersions by dynamic light scattering (DLS) showed an average particle diameter of $\sim 90 \mathrm{~nm}$ (Fig. 1h) and smaller aggregates (see Supplementary Information, Fig. S1) ascribed to micelles or PVA/SDS complexes ${ }^{14}$.

As OR has a low $\mathrm{CH}$ solubility $(<0.35 \mathrm{wt} \%)$, acetone co-solvent was added at low levels $(0.5 \mathrm{wt} \%)$ allowing dye 

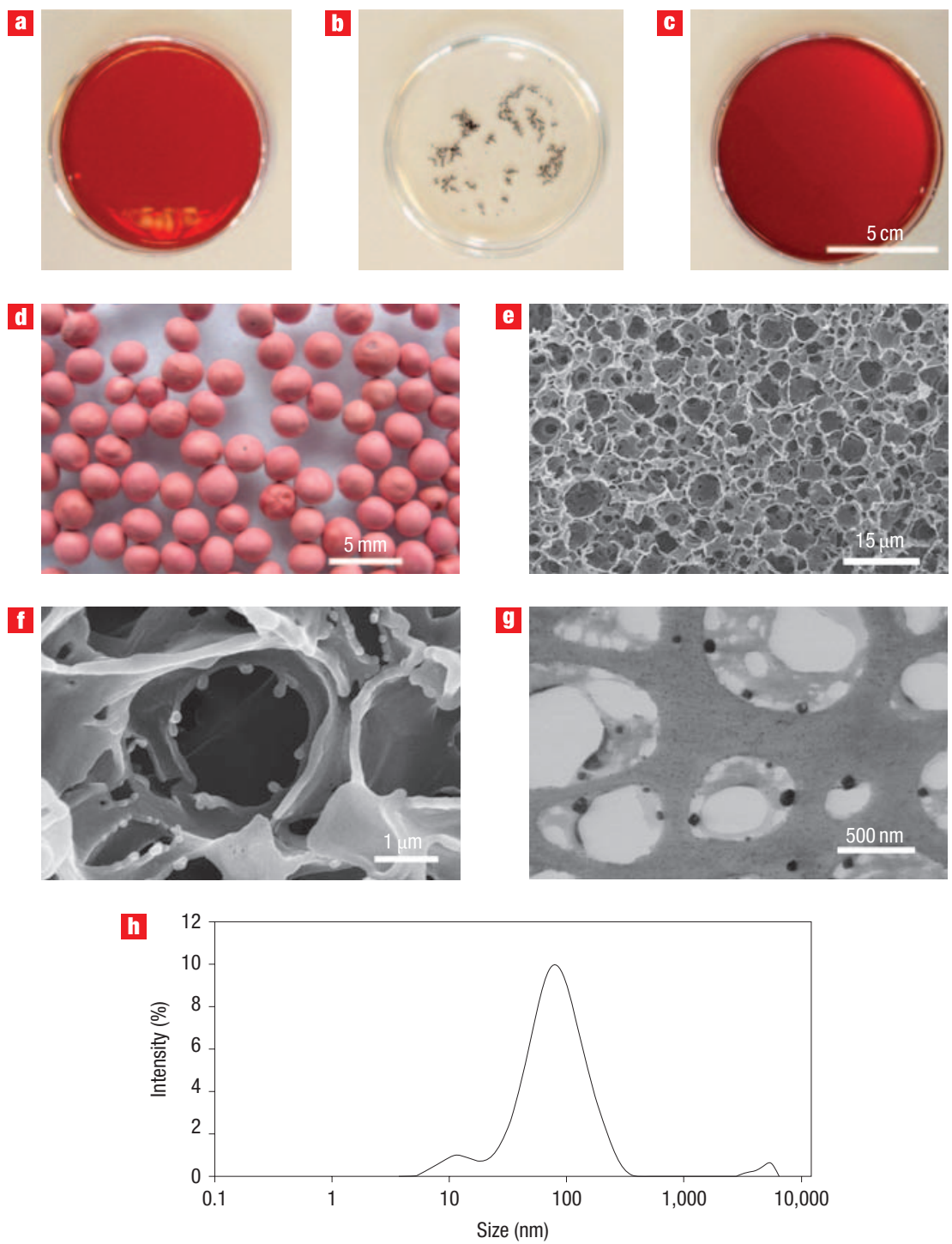

Figure 1 Characterization of solid nanocomposites and nanodispersions. a-c, Visual comparison of an aqueous nanodispersion of Oil-Red (OR) (a), OR powder added to water (b), and OR dissolved in acetone (c). d, Highly porous nanocomposite beads (poly(vinyl alcohol)/sodium dodecyl sulphate) containing $0 \mathrm{R}$ (0.018 wt\%). $\mathbf{e}$, SEM image of the internal porous bead structure. $\mathbf{f}$, Higher magnification showing nanoparticles associated with the edge of a pore. $\mathbf{g}$, STEM image of OR nanoparticles after drying the nanodispersion onto a porous carbon grid. $\mathbf{h}$, Dynamic light scattering analysis of the OR nanodispersion showing an average particle size of $90 \mathrm{~nm}$.

loadings up to $0.5 \mathrm{wt} \%$. SEM imaging (see Supplementary Information, Fig. S2) showed significantly more OR particles in the resulting composite. Acetone destabilized the emulsions somewhat, but rapid freezing still allowed porous emulsiontemplated structures. After the addition of water, STEM observations and DLS measurements (see Supplementary Information, Figs S3 and S4) showed significantly larger average particle diameters $(\sim 300 \mathrm{~nm})$.

OR can be solubilized in water by conventional PVA/SDS micellization. We therefore evaluated the potential of our system to disperse excess organic material to a greater extent than simple solubilization over various PVA/SDS ratios. All combinations were above their respective critical micelle concentrations (see Supplementary Information). The formation of OR-saturated micelles (SM) in water $\left(300 \mathrm{~h}\right.$ at $\left.20^{\circ} \mathrm{C}\right)$ was studied for each PVA/SDS ratio (Fig. 2a). These values form a baseline solubilization plot (Fig. 2b), representing the maximum OR concentration achievable at each ratio. For comparison, a series of emulsion-templated samples was produced with comparable SDS/PVA ratios and dispersed into water to produce OR nanodispersions (Fig. 2c). Nanodispersions with high stability $(<14$ days) (see Supplementary Information, Fig. S5) required both SDS and PVA (Fig. 2c). A sixfold increase in OR concentration $\left(6.5 \times 10^{-3} \mathrm{M}\right.$ OR, PVA $\left./ \mathrm{SDS}=7 \times 10^{-3} \mathrm{M} / 0.3 \mathrm{M}\right)$ was achieved over the saturated micelle concentration (SMC). Moreover, this was achieved only seconds after the addition of water, compared to extended SM equilibration times $(>300 \mathrm{~h}$ ). As the nanodispersions are formed almost instantly, they may be deployed at the point of use without requiring high-temperature, high-shear mixing or relying on molecular solubility. Higher loadings (more than $50 \times$ greater than the SMC) were achieved by further increasing the OR concentration in the oil phase; 


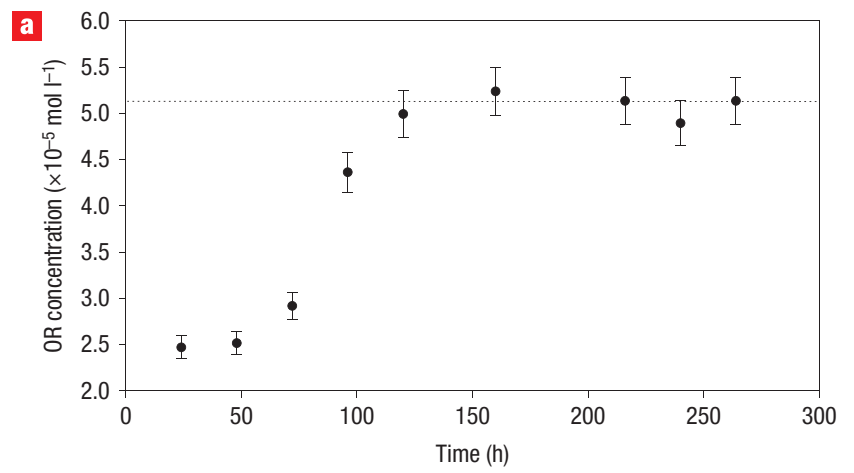

b

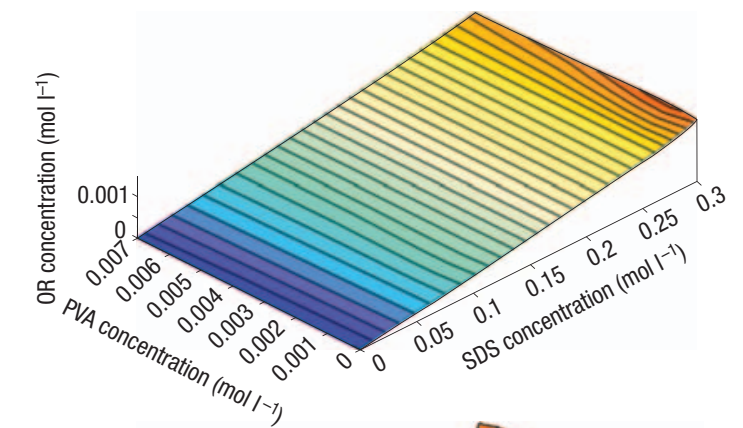

G

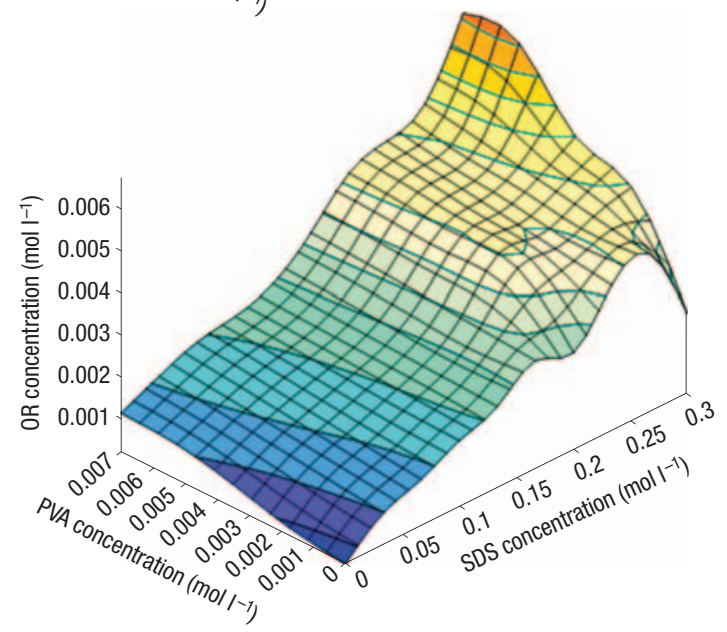

Figure 2 Comparison of conventional surfactant solubilization and nanodispersions of Oil-Red (OR). a, Saturation kinetics of OR in an aqueous micellar solution of SDS (SDS $=0.01 \mathrm{~mol} \mathrm{I}^{-1} ; \mathrm{PVA}=0$ ). The horizontal line indicates equilibration. $\mathbf{b}$, Baseline solubilization of $\mathrm{OR}$ using various SDS and PVA ratios. The plot represents maximum $O R$ solubilization within given polymer and surfactant concentrations. c, Aqueous concentration of $O R$ achieved using emulsion-templated nanodispersion technology (identical ratios of PVA and SDS to $\mathbf{b})$. The difference between $\mathbf{b}$ and $\mathbf{c}$ - the 'excess' concentration over the saturated micelles-indicates the presence of $\mathrm{OR}$ nanoparticles within the 'solution-like' dispersion.

however, larger OR particles $(>300 \mathrm{~nm})$ with reduced stability (2 days) were formed. Zeta-potential measurement (see Supplementary Information, Fig. S11) showed negatively charged nanoparticles, indicating SDS surface adsorption (low OR loading $=-23.3 \pm 1.2 \mathrm{mV}$; high OR loading $=-21.8 \pm 0.188 \mathrm{mV}$ ).

The Horn and Rieger ${ }^{4}$ categories have several common features. Apart from precipitation, the methods use mobile/liquid emulsions containing water-insoluble compounds dissolved within the oil phase. As the oil is slowly, controllably and selectively removed, the oil droplets shrink, and the solute concentrates and ultimately solidifies. Our technique solidifies the entire emulsion by freezing to form static oil droplets. Whole-droplet motion is prevented during the simultaneous sublimation of both frozen water and solvent. In contrast to other techniques, the oil droplets are depleted of oil in the presence of a static surface structure or support.

The mechanism of nanoparticle formation is complex; however, the processes operating during conventional freeze-drying of aqueous solutions are well understood ${ }^{1,15-17}$. Initial freezing generates ice crystals of pure water ${ }^{18}$, leading to dramatic concentration and viscosity increases within the remaining liquid ${ }^{1}$. The resulting desiccation is highly efficient ( $>99 \%$ water removal from the solute) and causes phase separation ${ }^{15}$. Crystallization of the concentrated liquid regions is viscosity-inhibited ${ }^{17}$, and final solidification produces crystalline, amorphous or mixed solids. Supercooling is at its highest when liquid nitrogen is used to freeze small volumes ${ }^{1,15}$, leading to significant liquid stresses ${ }^{16}$. Our technique involves the freezing/freeze-drying of emulsions (volumes ranging from $\sim 6 \times 10^{-8} \mathrm{~cm}^{3}$ for atomization to $\sim 2 \mathrm{~cm}^{3}$ for direct freezing) and similar concentration of the solute in the organic phase occurs ${ }^{18}$. The individual, isolated oil droplets have very small volumes $\left(\sim 5 \times 10^{-10} \mathrm{~cm}^{3}\right.$ for beads) and undergo significant local supercooling and concentration during freezing (Fig. 3b). Areas of saturated solute will develop that are subject to precipitation/crystallization during water/oil removal (Fig. 3c). Particles thus formed will be dispersed upon addition of water and dissolution of the solid porous polymer/surfactant support. PVA is a known dispersion stabilizer, binding extremely strongly to nanoparticle surfaces ${ }^{1}$ and contributing to dispersion stability (Fig. 3d).

The formation of dry, redispersible solids containing nanoparticles offers significant advantages over liquid-dispersion storage and transport. OR/PVA beads formed identical clear, red nanodispersions after two years without stringent storage conditions. As a result of using rapid freezing, emulsion stability is not critical and the choice of surfactants, hydrophilic polymers, solvents and organic active ingredients is therefore very broad, including biodegradable polymers and temperature-sensitive drugs. Nanoparticle loadings were adjusted by changing the $\mathrm{O} / \mathrm{W}$ ratio with available oil-phase fractions from 0.01 to at least 0.9 . Porosity (Fig. $4 \mathrm{a}-\mathrm{c}$ ), dissolution kinetics and material properties are also tuneable by means of this mechanism, in contrast to the reported spray-freezing-into-liquid processes for organic microparticle preparation ${ }^{19,20}$.

We have produced porous surfactant/silica powders (Fig. $4 \mathrm{e}-\mathrm{f}$ ) with our process, avoiding spray pyrolysis or templating/etching routes $^{21-23}$. Also, to remove the reliance on volatile organic solvents, we have recently adopted liquid $\mathrm{CO}_{2}$ (refs 24-28) as the emulsion oil phase. After directly freeze-drying the $\mathrm{CO}_{2}$ /water emulsions, porous polymer monoliths that dissolve rapidly in water and form clear OR nanodispersions (diameter $\sim 240 \mathrm{~nm}$ ) were formed (see Supplementary Information, Fig. S9).

The practical value of the approach was demonstrated by forming aqueous nanodispersions of poorly soluble commercial organic biocides, which are typically used in products ranging from cosmetics to agrochemicals. For example, Triclosan (5-chloro-2-(2,4-dichlorophenoxy)phenol) has low watersolubility $\left(10 \mu \mathrm{g} \mathrm{ml}^{-1}\right)$ and limited biocidal activity without the use of high doses, co-solvents or oil phases ${ }^{29}$. Also, micellization is known to decrease bactericidal activity ${ }^{29}$. The minimum inhibitory concentration $\left(\mathrm{MIC}_{50}\right)$ against the Gram-negative bacterium Corynebacterium of an ethanol/water solution of Triclosan/PVA/SDS was compared with the $\mathrm{MIC}_{50}$ of aqueous nanodispersions prepared by our route. 


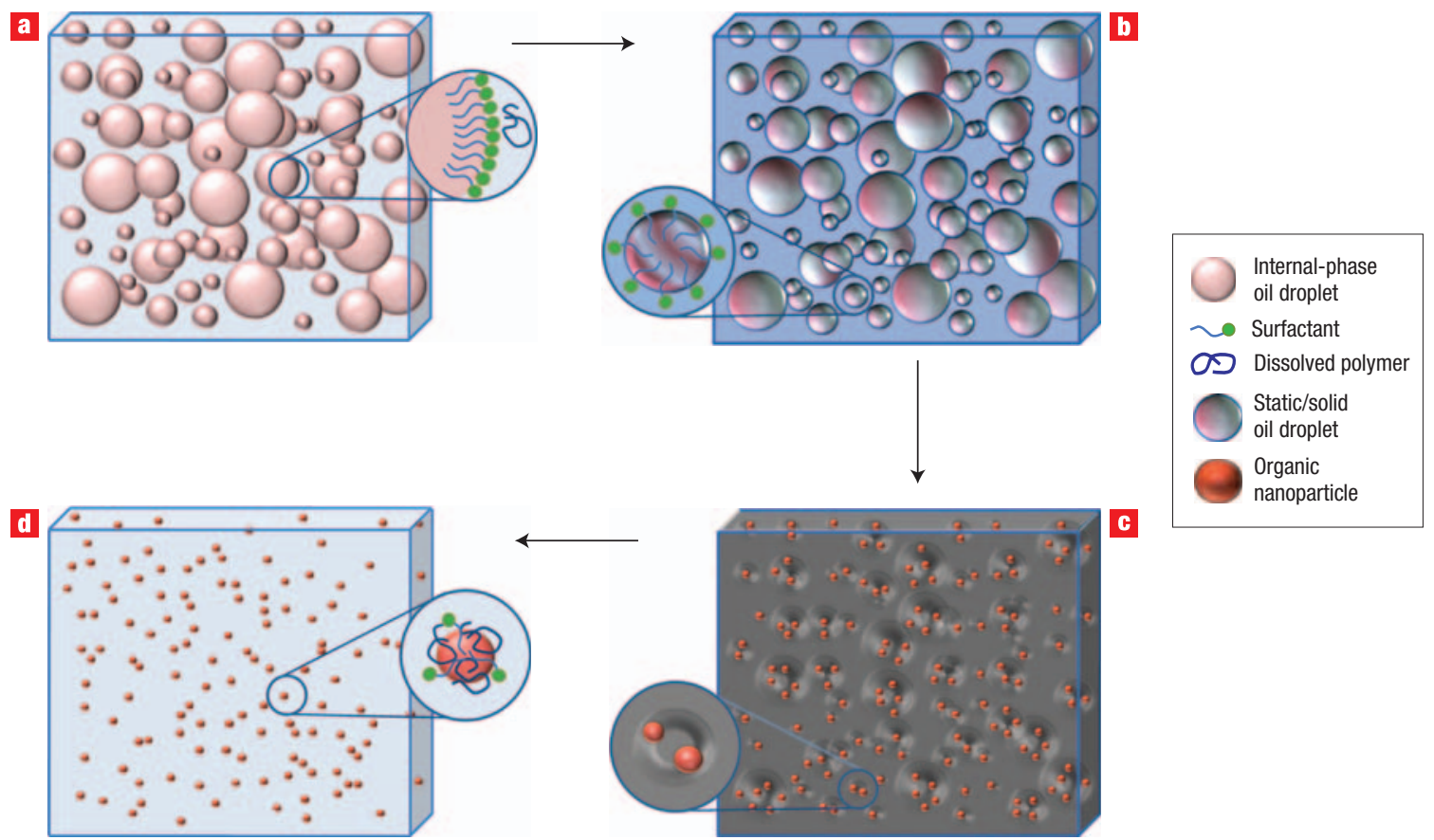

Figure 3 Schematic/mechanistic representation of nanoparticle formation during freeze-drying. a, Oil-in-water emulsion; organic compound dissolved in internal-phase oil droplets. Enlarged area: homogeneous, surfactant-stabilized oil droplet and dissolved polymer. b, Frozen oil-in-water emulsion; static/solid oil droplets within a solidified continuous phase. Enlarged area: organic compound inhomogeneously distributed within the frozen oil droplet and associated surfactant. c. Emulsion-templated porous solid after freeze-drying. Enlarged area: organic nanoparticles within open pores. d, Organic nanodispersion after addition of water. Enlarged area: surfactant- and polymer-stabilized organic nanoparticle.
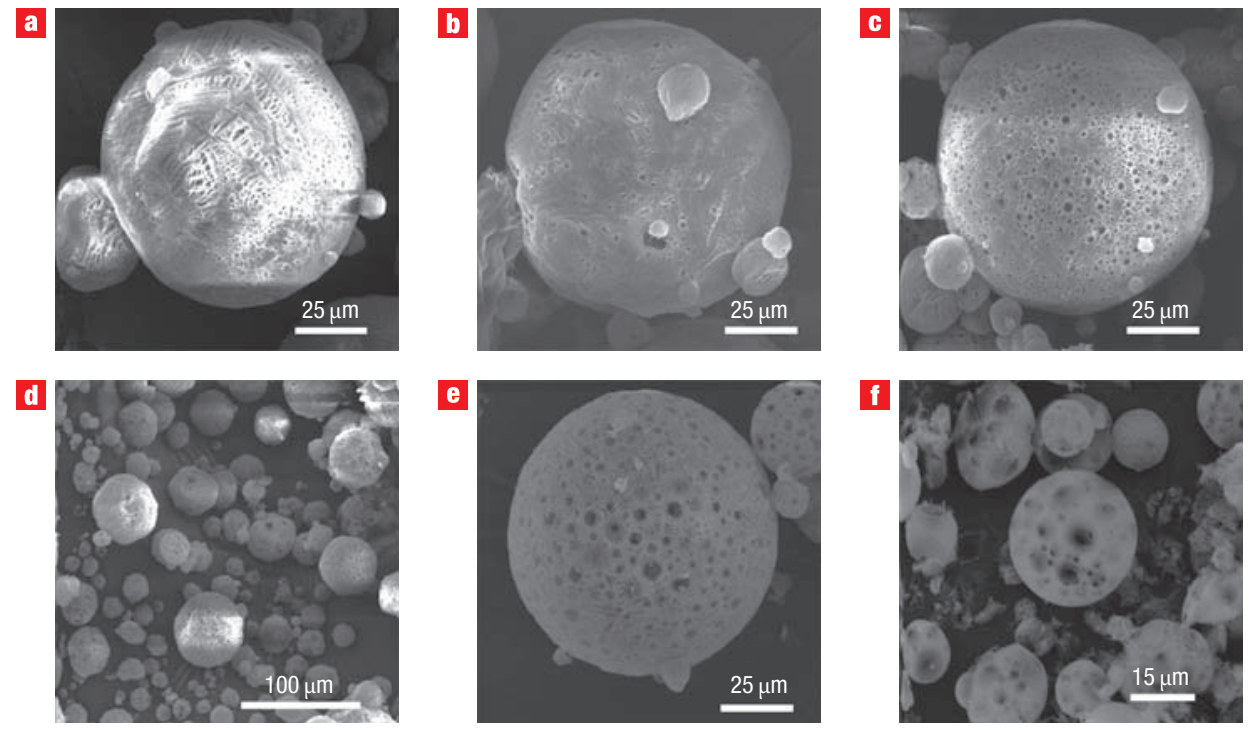

Figure 4 Scanning electron microscopy images of freeze-dried atomized powders. a-c, Freeze-dried PVA/SDS powders with $0 \%$ emulsion-templating (a), $20 \%$ emulsion-templating (b), and 50\% emulsion-templating (c). d, Low-magnification image of powder with $50 \%$ emulsion-templating. e, Emulsion-templated powder containing SDS only (66.7\% emulsion-templating). f, Emulsion-templated silica powder after calcination; original emulsion contained PVA and silica HS-30 (75\% emulsion-templating).

The ethanol/water system showed substantial inhibition of regrowth of the bacterium at Triclosan concentrations $>100$ p.p.m. (Fig. 5a). Inhibition was lost rapidly at concentrations $<100$ p.p.m., with an observed $\mathrm{MIC}_{50}$ of approximately 50 p.p.m. In contrast, aqueous nanodispersions were significantly more active $(2.5 \%$ regrowth at 50 p.p.m.), with bacterial regrowth inhibited to $<50 \%$ at 6.25 p.p.m. (Fig. 5b). In both cases, 100\% regrowth of Corynebacterium was observed between 1.56 and 0.78 p.p.m., 

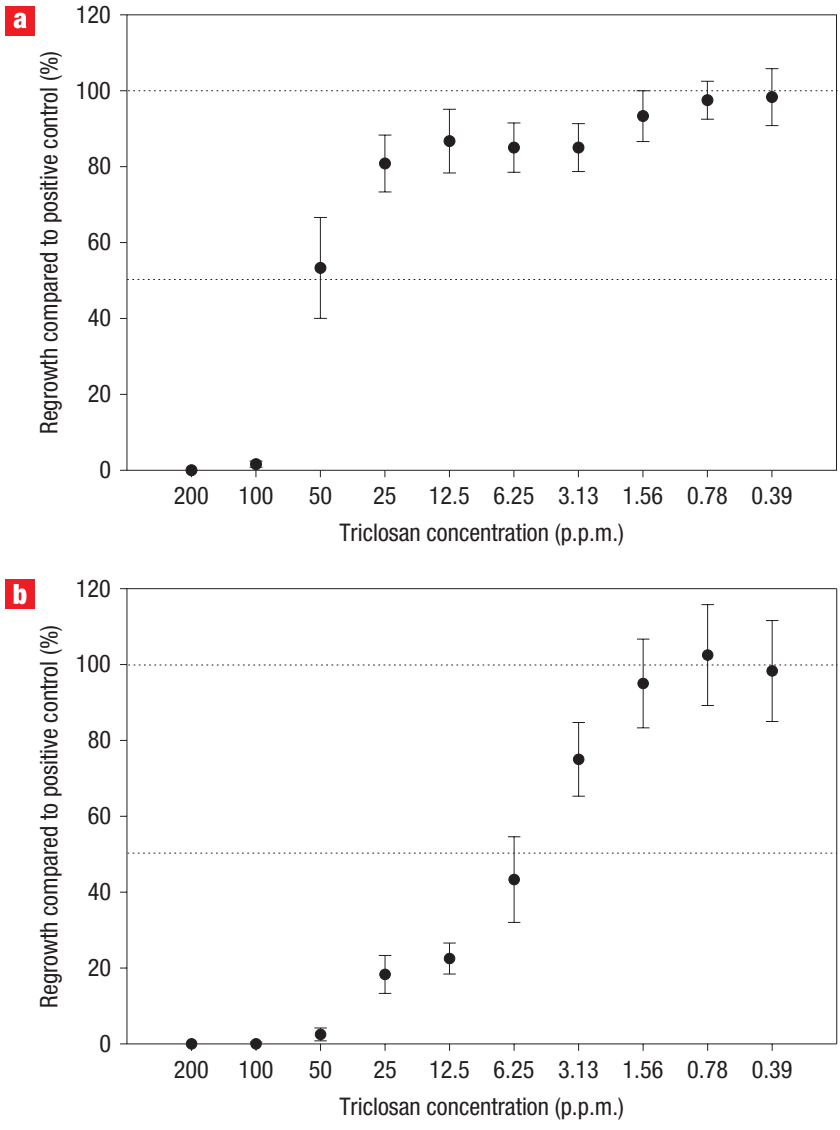

Figure 5 Evaluation of the minimum inhibitory concentration (MIC) of different forms of the antibacterial agent Triclosan. $a, b$, Regrowth profiles of the Gram-positive bacterium Corynebacterium in the presence of an aqueous/ethanol solution of Triclosan/SDS/PVA (a), and aqueous Triclosan nanodispersion with SDS and PVA present (b). The horizontal lines depict the $\mathrm{MIC}_{50}$ (lower) and complete regrowth (upper) relative to positive control. Error bars are derived from ten repeat measurements. All measurements were conducted in a brain/heart infusion.

suggesting similar biocidal mechanisms at low concentrations and possibly indicating nanoparticle dissolution at these concentrations. The observed $\mathrm{MIC}_{50}$ reduction (one-eighth of the solution $\mathrm{MIC}_{50}$ ) without the use of co-solvents is of clear interest across many applications and our ongoing experiments are focused on establishing the mode of action.

To summarize, we have demonstrated the generic formation of organic nanodispersions by freeze-drying emulsions containing water-insoluble compounds dissolved in a volatile oil phase. Water-soluble materials present in the aqueous phase form a porous solid support that dissolves rapidly on addition of water to disperse the nanoparticles. This technique has wide applicability, because nanodispersions are a valuable alternative to molecular solutions. Water-insoluble commercial biocides showed increased activity, suggesting the potential for decreased application doses and lower rates of acquired bacterial resistance.

\section{METHODS}

VARIATION OF POROSITY BY MEANS OF INTERNAL-PHASE VOLUME CONTROL

A range of 'unloaded' porous PVA/SDS materials were produced using oil-phase fractions of 0, 0.2, 0.4, 0.6 and 0.75 (see Supplementary Information,
Fig. S6) to demonstrate the control of porosity using our technique. Materials produced with no oil phase present showed porosity derived solely from ice-templated macropores, formed by phase separation during the freezing process $^{18}$. As the $\mathrm{O} / \mathrm{W}$ ratio increases, the number of emulsion-templated pores increases accordingly. At an internal-phase fraction of $\sim 0.75$, the porosity is derived solely from the emulsion-templating process, as supported by pore volume measurements (see Supplementary Information, Fig. S7). Materials with larger pore volumes were found to dissolve more rapidly than those with lower pore volumes and hence the dissolution kinetics can be fine-tuned (see Supplementary Information, Figs S7 and S8).

\section{$\mathrm{CO}_{2}$ METHODS}

$\mathrm{CO}_{2}$-in-water emulsions, containing $\mathrm{OR}$ dissolved in the liquid $\mathrm{CO}_{2}$ phase and PVA in the aqueous phase, were frozen by liquid nitrogen immersion followed by freeze-drying (see Supplementary Information). The emulsions were stabilized by an inexpensive triblock copolymer, poly(vinyl acetate)- $b$-polyethylene glycol$b$-poly(vinyl acetate), which was synthesized as reported earlier ${ }^{28}$.

\section{MIC $_{50}$ EVALUATION}

Biocidal activity was measured against a Gram-negative bacterium, Corynebacterium, in a standard growth medium, and regrowth was compared against positive controls. Triclosan was either dissolved into an aqueous brain/heart infusion (BHI) containing 30\% ethanol or dispersed into BHI with no additional solvent to form initial concentrations of 400 p.p.m. (PVA and SDS were also present in the $\mathrm{BHI} /$ ethanol comparison to match the nanocomposite composition: Triclosan/SDS/PVA $=10 / 63 / 27 \mathrm{wt} \%$ ). Subsequent dilution with either $\mathrm{BHI} /$ ethanol or $\mathrm{BHI}$ alone generated a series of lower concentrations, which were inoculated with Corynebacterium. After plating into wells, the samples were incubated at $37^{\circ} \mathrm{C}$ for $18 \mathrm{~h}$. All measurements were repeated ten times to ensure significance. $\mathrm{MIC}_{50}$ values were assessed as the concentration required to maintain $<50 \%$ bacterial regrowth relative to the control. All control experiments included SDS and PVA and additional ethanol as required.

Received 30 January 2008; accepted 9 June 2008; published 6 July 2008.

\section{References}

1. Abdelwahed, W., Degobert, G., Stainmesse, S. \& Fessi, H. Freeze-drying of nanoparticles: Formulation, process and storage considerations. Adv. Drug Deliv. Rev. 58, $1688-1713(2006)$

2. Mizoe, T., Ozeki, T. \& Okada, H. Preparation of drug nanoparticle-containing microparticles using a 4-fluid nozzle spray drier for oral, pulmonary and injection dosage forms. J. Contr. Rel. 122, $10-15$ (2007).

3. Texter, J. Precipitation and condensation of organic particles. J. Disp. Sci. Technol. 22, 499-527 (2001).

4. Horn, D. \& Rieger, J. Organic nanoparticles in the aqueous phase-theory, experiment and use. Angew. Chem. Int. Ed. Engl. 40, 4330-4361 (2001).

5. Zhang, H. \& Cooper, A. I. Synthesis and applications of emulsion-templated porous materials. Soft Matter 1, 107-113 (2005).

6. Royal Society and Royal Academy of Engineering Nanoscience and Nanotechnologies. Nanoscience and Nanotechnologies: Opportunities and Uncertainties, Report (Royal Society and Royal Academy of Engineering Nanoscience and Nanotechnologies, 2004) < http://www.nanotec.org.uk/finalReport.htm > .

7. Rabinow, B. E. Nanosuspensions in drug delivery. Nature Rev. Drug Discov. 3, 785-796 (2004).

8. Galindo-Rodríguez, S. A. et al. Comparative scale-up of three methods for producing ibuprofenloaded nanoparticles. Eur. J. Pharm. Sci. 25, 357-367 (2005).

9. Lee, J. C. et al. Preparation, stability and in vitro performance of vesicles made with diblock copolymers. Biotechnol. Bioeng. 73, 135-145 (2001).

10. Sethi, V., Önyüksel, H. \& Rubinstein, I. Liposomal vasoactive intestinal peptide. Method. Enzymol. 391, 377-395 (2005)

11. Kita-Tokarczyk, K., Grumelard, J., Haefele, T. \& Meier, W. Block copolymer vesicles — using concepts from polymer chemistry to mimic biomembranes. Polymer 46, 3540-3563 (2005).

12. Cameron, N. R. High internal phase emulsion templating as a route to well-defined porous polymers. Polymer 46, 1439-1449 (2005).

13. Dwivedi, A. M. Residual solvent analysis in pharmaceuticals. Pharm. Technol. Eur. 14, 26-28 (2002).

14. Gilanyi, T. \& Wolfram, E. Interaction of ionic surfactants with polymers in aqueous solution. Coll. Surf. 3, 181-198 (1981).

15. Tang, X. \& Pikal, M. J. Design of freeze-drying processes for pharmaceuticals: practical advice. Pharm. Res. 21, 191-200 (2004).

16. Franks, F. Freeze-drying of bioproducts: putting principles into practice. Eur. J. Pharm. Biopharm. 45, 221-229 (1998)

17. Craig, D. Q. M., Royall, P. G., Kett, V. L. \& Hopton, M. L. The relevance of the amorphous state to pharmaceutical dosage forms: glassy drugs and freeze-dried systems. Int. J. Pharm. 179, 179-207 (1999).

18. Zhang, H. et al. Aligned two- and three-dimensional structures by directional freezing of polymers and nanoparticles. Nature Mater. 4, 787-793 (2005).

19. Yu, Z., Rogers, T. L., Hu, J., Johnston, K. P. \& Williams, R. O. Preparation and characterization of microparticles containing peptide produced by a novel process: spray freezing into liquid. Eur. J. Pharm. Biopharm. 54, 221-228 (2002)

20. Rogers, T. L. et al. Micronized powders of a poorly water soluble drug produced by a spray-freezing into liquid-emulsion process. Eur. J. Pharm. Biopharm. 55, 161-172 (2003).

21. Skrabalak, S. E. \& Suslick, K. S. Porous $\mathrm{MoS}_{2}$ synthesized by ultrasonic spray pyrolysis. J. Am. Chem. Soc. 127, 9990-9991 (2005). 
22. Iskandar, F., Mikrajuddin \& Okuyama, K. Controllability of pore size and porosity on self-organized porous silica particles. Nano Lett. 2, 389-392 (2002)

23. Suh, W. H. \& Suslick, K. S. Magnetic and porous nanospheres from ultrasonic spray pyrolysis. J. Am Chem. Soc. 127, 12007-12010 (2005)

24. DeSimone, J. M. Practical approaches to green solvents. Science 297, 799-803 (2002).

25. Cooper, A. I. Porous materials and supercritical fluids. Adv. Mater. 15, 1049-1059 (2003).

26. Zhang, H., Long, J. \& Cooper, A. I. Aligned porous materials by directional freezing of solutions in liquid $\mathrm{CO}_{2}$. J. Am. Chem. Soc. 127, 13482-13483 (2005).

27. Butler, R., Hopkinson, I. \& Cooper, A. I. Synthesis of porous emulsion-templated polymers using high internal phase $\mathrm{CO}_{2}$-in-water emulsions. J. Am. Chem. Soc. 125, 14473-14481 (2003).

28. Tan, B. \& Cooper, A. I. Functional oligo(vinyl acetate) $\mathrm{CO}_{2}$-philes for solubilization and emulsification. J. Am. Chem. Soc. 127, 8938-8939 (2005).

29. Taylor, T. J. et al. Physicochemical factors affecting the rapid bactericidal efficacy of the phenolic antibacterial triclosan. Int. J. Cosmetic Sci. 26, 111-116 (2004).

Supplementary Information accompanies this paper at www.nature.com/naturenanotechnology.

\section{Acknowledgements}

The authors thank IOTA NanoSolutions Limited for an Industrial Senior Research Fellowship (S.R.), the Royal Society for a University Research Fellowship (A.C.) and an Industry Fellowship (S.R.) and Research Councils UK for an Academic Fellowship (H.Z.). We also acknowledge the Centre for Materials Discovery (University of Liverpool) for access to analytical equipment and specifically J. Weaver for help conducting surface tension measurements. The EPSRC (grant GR/N39999/01 and Portfolio Partnership in Complex Materials Discovery EP/C511794/1), IOTA NanoSolutions Limited and Unilever are thanked for Materials Discovery
financial support.

Author contributions

All authors contributed to the experimental programme, either through design of the experiment or practice. D.T. led the microbiological testing. H.Z., A.C. and S.R. co-wrote the manuscript.

\section{Author information}

Reprints and permission information is available online at http://npg.nature.com/reprintsandpermissions/. Correspondence and requests for materials should be addressed to A.I.C. and S.P.R. 